\title{
Schutzverhalten von weichballistischem ultrahochmolekularem Polyethylen
}

\author{
Michael Hendrix*, Michael Herzog
}

\section{Zusammenfassung}

Diese Publikation befasst sich mit dem Langzeitverhalten der Schutzeigenschaften von weichballistischem ultrahochmolekularem Polyethylen. Vorrangig soll die Frage geklärt werden, ob dieses Material qualitätsmindernde Erscheinungen im Schutzverhalten aufweist, die mit seinem fortschreitenden Alter einhergehen. Um diese Frage zu klären, wurden anhand zahlreicher Beschussversuche an polizeilichen Schutzwesten und Prüfmustern statistisch verlässliche Daten gesammelt und anschließend unter verschiedenen Blickwinkeln ausgewertet. Ein wesentliches Ergebnis der Untersuchungen ist, dass sich die Schutzeigenschaften von weichballistischem ultrahochmolekularem Polyethylen zumindest über einen Zeitraum von 30 Jahren nicht nennenswert ändern.

\section{Abstract}

This publication deals with the long-term behavior of the ballistic protection of ultra high molecular weight polyethylene. It aims to answer the question, whether ballistic material shows significant signs of a deterioration that comes with preceding age. To answer that question numerous shooting tests with bulletproof vests and test samples were made to collect statistically reliable data. The data then have been evaluated from different point of views. An essential result of the tests is that no appreciable deterioration of the ballistic protection can be recognized with preceding age up to 30 years.

\section{Einführung}

Schutzsysteme aus weichballistischen Materialien spielen in vielen Lebensbereichen aufgrund ihres geringen Gewichts bei hohem Schutz eine immer größer werdende Rolle. Basis sind zumeist Hochleistungsfasern, die auch für Seile und Gurte im Sport- und Freizeitbereich oder für Faserverbundwerkstoffe genutzt werden. Ein bekanntes Beispiel eines ballistischen Schutzsystems sind polizeiliche Schutzwesten (siehe $z$. B. Technische Richtlinie „Ballistische Schutzwesten“ aus dem Jahr 2008 (Polizeitechnisches Institut der Deutschen Hochschule der Polizei 2008)). Materialien für weichballistische Anwendungen spielen aber nicht nur als wesentlicher Bestandteil von Schutzkleidung eine Rolle, sondern werden zum Beispiel auch für den hartballistischen Schutz zur Panzerung von Fahrzeugen und in zunehmendem Maße auch zur passiven Gefahrenab- wehr bei terroristischen Anschlägen eingesetzt (siehe z. B. Prüfung von ballistischem Material für Sonderschutzfahrzeuge bei Frieß 2006).

Die Wirkungsweise von weichballistischen Materialien lässt sich grob folgendermaßen beschreiben: Wenn ein Geschoss auf eine mehrlagige Struktur aus reißfestem (weichballistischem) Gewebe oder Gelege trifft, wird die kinetische Energie des Geschosses abgebaut, indem das Geschoss beim Durchdringen der einzelnen Gewebe- bzw. Gelegelagen diese zerstört oder dehnt, wobei auch am Geschoss (je nach Typ z. T.) beträchtliche Verformungsarbeit geleistet wird. Nach dem Durchdringen mehrerer Lagen hat das Geschoss seine kinetische Energie vollständig abgegeben und bleibt stecken. Weichballistische Materialien werden hauptsächlich zum Schutz vor Kurzwaffengeschossen oder beschleunigten Metallsplittern eingesetzt.
Schutzsysteme aus weichballistischen Materialien enthalten als wirksame Komponente zumeist Gewebe aus hochfesten synthetischen Fasern auf der Basis aromatischer Polyamide (Aramide) oder Gelege auf der Basis von ultra-hochmolekularem Polyethylen (UHMW-PE). Das Langzeitverhalten von Aramid-Geweben hatten wir schon vor einiger Zeit analysiert (Geßner \& Hendrix 2014). Im Folgenden sollen die Ergebnisse systematisch durchgeführter Langzeituntersuchungen an PolyethylenGelegen dargestellt werden. Zur Klärung der stofflichen Mechanismen, die einer möglichen altersbedingten Änderung der Schutzwirkung von ultra-hochmolekularem Polyethylen zugrunde liegen, wurden Beschussversuche an Prüfmustern und polizeilichen Schutzwesten durchgeführt. Umfassend untersucht wurden Schutzwesten und Prüfmuster unterschiedlichen Alters, hergestellt aus dem Gelege SB21 und dem Gelege 
SB115. Beide Gelege sind UHMWPE UD (ultra high molecular weight polyethylene unidirectional) von DSM Dyneema ${ }^{\circledast}$. DSM produziert das UHMW-PE-Pulver, die Faser und das Gelege selbst. Dyneema ${ }^{\circledast}$ ist ein eingetragenes Warenzeichen der Royal DSM N.V. Der prinzipielle Versuchsaufbau für die Beschussversuche ist der VPAM APR 2006 zu entnehmen (Vereinigung der Prüfstellen für angriffshemmende Materialien und Konstruktionen 2010: 28).

Die Westen-Einschübe waren mit 34 Lagen SB21 bzW. 46 Lagen SB115 ausgestattet - die getragenen Westen können je nach Produktspezifikation der Westenhersteller zum Zeitpunkt der Herstellung eine abweichende Anzahl von Lagen aufweisen (die Lagenanzahl wurde manuell überprüft). Einschübe mit 34 Lagen SB21 waren eine Entwicklung für die Schutzklasse SK 1 nach der Technischen Richtlinie Schutzwesten aus dem Jahr 2000 (Polizeitechnisches Institut der Deutschen Hochschule der Polizei), Einschübe mit 46 Lagen SB115 waren eine Entwicklung für die Schutzklasse SK 1 nach der Technischen Richtlinie Schutzwesten aus dem Jahr 2008 (Polizeitechnisches Institut der Deutschen Hochschule der Polizei).

\section{Künstliche Alterung}

Die künstliche Alterung von 40×40 cm-Prüfmustern aus SB21-Gelegen und aus SB115-Gelegen wurde durchgeführt, indem die Prüfmuster eine Zeit lang hängend im Wärmeschrank bei einer konstanten Temperatur von $75^{\circ} \mathrm{C}$ und Gleichgewichtsluftfeuchtigkeit aufbewahrt wurden. Das entsprechend erreichte Alter hängt von der Verweildauer im Wärmeschrank ab und lässt sich für SB21 dem Arrhenius-Plot aus Fig. 2 der Publikation von Chabba et al. 2007 entnehmen. Legt man denselben Alterungsmechanismus zugrunde wozu es aufgrund der chemischen Struktur keine Vorbehalte gibt -, kann dieser Arrhenius-Plot auch für SB115 verwendet werden.

Insgesamt wurden 8 Prüfmuster aus SB21 und 8 Prüfmuster aus SB115
Tab. 1: Das Gelege SB21 basiert auf dem Garn SK76, das Gelege SB115 auf dem Garn SK99. Nachfolgend sind einige Parameter dieser Garne und des entsprechenden Geleges angeführt (DSM Dyneema 2017):

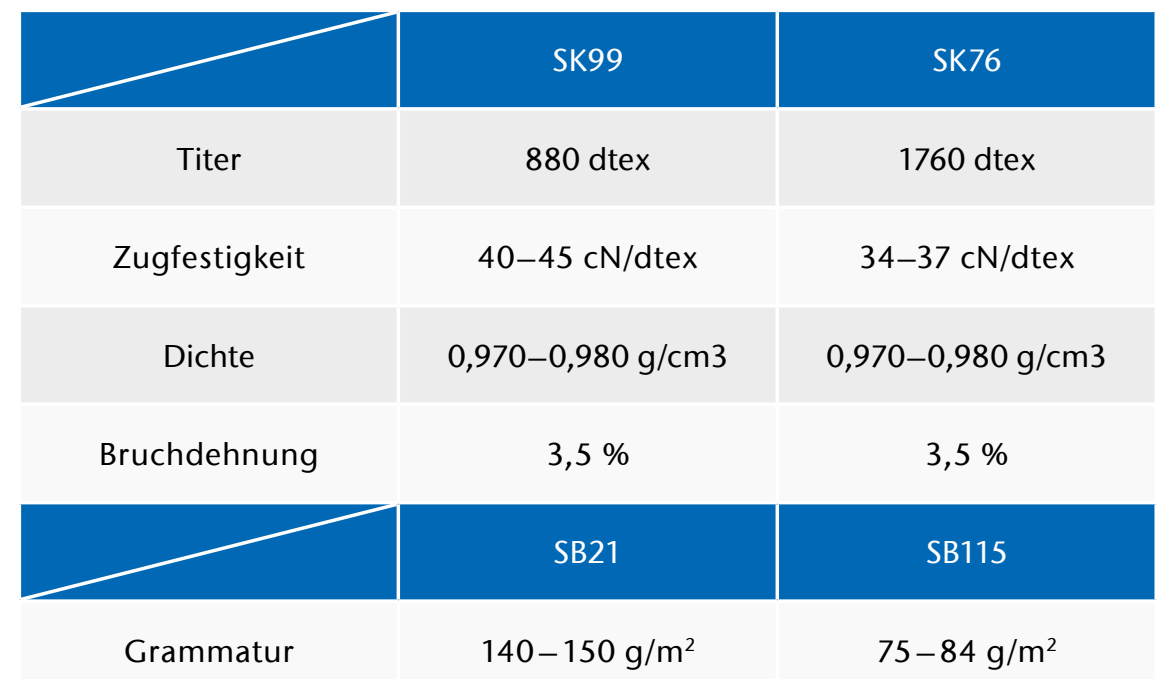

künstlich gealtert, wobei jeweils 4 Prüfmuster 8 Wochen lang und 4 Prüfmuster 16 Wochen lang im Wärmeschrank verweilten. Dem Arrhenius-Plot ist zu entnehmen, dass einer Verweildauer von 8 Wochen bei $75^{\circ} \mathrm{C}$ ein simuliertes Alter von 15 Jahren, einer Verweildauer von 16 Wochen bei $75^{\circ} \mathrm{C}$ ein simuliertes Alter von 30 Jahren entspricht.

\section{Beschussversuche an Westen und Prüfmustern}

\subsection{Vorbereitende Arbeiten}

Bevor die uns zur Verfügung stehenden älteren (schon getragenen) Schutzwesten beschossen wurden, wurden die Westen auf Beschädigungen hin untersucht, die Lagen gezählt und weitere relevante Parameter ermittelt. Für die dann wirklich beschossenen Westen sind noch zusätzlich die RAL-Farbwerte sowie der Beschussort (Mellrichstadt oder DSM) angegeben. Die Schutzwesten wurden in der Schutzhülle, aber ohne Schockabsorber, beschossen.

Zusätzlich zu den Schutzwesten wurden auch Prüfmuster mit den Maßen $40 \times 40 \mathrm{~cm}$ beschossen. Die Prüfmuster wurden teilweise künstlich gealtert: "8 Wochen bei $75^{\circ} \mathrm{C}$ gelagert" entspricht einem simulierten Alter von 15, , 16 Wochen bei $75^{\circ} \mathrm{C}$ gelagert" einem simulierten Alter von 30 Jahren.
Gelege aus ultra-hochmolekularem Polyethylen ändern mit zunehmendem Alter ihre Farbe. Nach Informationen von DSM Dyneema wird diese Farbänderung durch die Matrix und nicht durch das Garn verursacht. Neuwertige Gelege haben einen RAL-Wert von 9003, was der Farbe "Signalweiß" entspricht. Mit zunehmendem Alter geht die Farbe ins Gelbliche über. Zum Beispiel ist eine 15 Jahre alte Schutzweste, hergestellt aus SB21, etwas gelber als reinweiß (Letzteres entspricht dem RAL-Wert 9010).

Da auch Prüfmuster aus dem Gelege SB115 untersucht wurden (SB115 ist ein recht neues Produkt, die Markteinführung erfolgte 2012), war man darauf angewiesen, künstlich zu altern, da entsprechend natürlich gealtertes Material noch nicht vorliegt. Unter Verwendung des vorliegenden Arrhenius-Plots und gestützt durch den Vergleich der künstlich und natürlich gealterten Westen aus SB21 sollte die künstliche Alterung der natürlichen Alterung weitgehend entsprechen. Zur Absicherung wurden auch Prüfmuster aus SB21 künstlich gealtert und anhand des RAL-Wertes mit entsprechend natürlich gealterten Schutzwesten verglichen. Bei allen 15 Jahre künstlich gealterten Prüfmustern aus SB21 ist die Farbe sehr ähnlich den entsprechend natürlich gealterten Schutzwesten (etwas gelber als reinweiß). Bei einem 
30 Jahre künstlich gealterten Prüfmuster aus SB21 ist der RAL-Wert 1015, was der Farbe „Hellelfenbein" entspricht. Zusätzlich ist bei 30 Jahre künstlich gealterten Gelegen aus SB21 auf der Prüfmusteroberfläche eine größere Anzahl von maisgelben Spots zu beobachten.

Die Farben für Gelege aus SB115 sind die folgenden:

\section{Jahre alt 9003 Signalweiß \\ 15 Jahre alt 9010 Reinweiß \\ 30 Jahre alt etwas heller als 1013 (Perlweiß)}

Für die Beschussversuche bei DSM wurde als Plastilin Caran d'Ache benutzt. Als Geschosse wurden verwendet: $9 \mathrm{~mm}$, VM-Sintox, $8 \mathrm{~g}$, verzinnt aus der Charge 10535GESCH.

\subsection{Beschussversuche an Westen und Prüfmustern aus SB21}

Ein Maß zur Bestimmung der Schutzeigenschaften eines ballistischen Schutzsystems ist die Bestimmung der V50-Grenzgeschwindigkeit. Dieser V50-Wert ist die Geschwindigkeit eines Geschosses, bei der die Wahrscheinlichkeit eines Durchschusses bei $50 \%$ liegt. Zur Berechnung des Wertes gibt es unterschiedliche Methoden. Im Rahmen der in diesem Kapitel geschilderten Untersuchungen wurden die Beschusstests nach STANAG 2920 (NATO Standardization Agency 2003) durchgeführt. Dabei wird das ballistische Paket an maximal 12 fest definierten Positionen auf dem Paket beschossen. Die V50-Grenzgeschwindigkeit berechnet sich aus dem arithmetischen Mittel von drei Durch- und drei Steckschüssen, wobei allerdings die Differenz zwischen der höchsten und der niedrigsten dieser sechs Geschossgeschwindigkeiten nicht mehr als $40 \mathrm{~m} / \mathrm{s}$ betragen darf. Als weitere Regel kommt hinzu, dass der Durchschuss mit der niedrigsten und der Steckschuss mit der höchsten Geschwindigkeit auf jeden Fall mit in die Berechnung des V50-Wertes einzubeziehen sind.

Die Abb. 1 zeigt den nach STANAG 2920 ermittelten Wert der V50-Grenzgeschwindigkeit für eine größere Anzahl von Schutzwesten und Prüf-

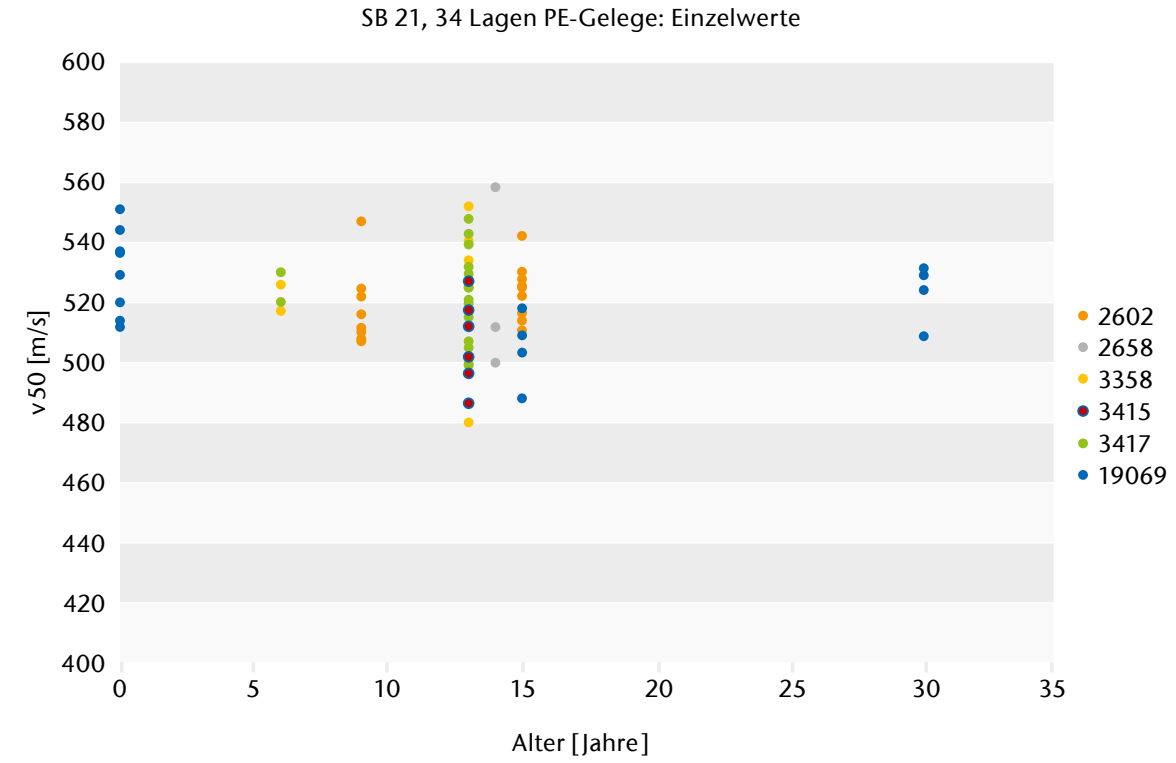

Abb. 1) V50-Werte von unterschiedlichen Produktionschargen aus SB21 in Abhängigkeit vom Alter. Die Standardabweichung für die V50-Werte liegt in der Größenordnung 10 bis $15 \mathrm{~m} / \mathrm{s}$.

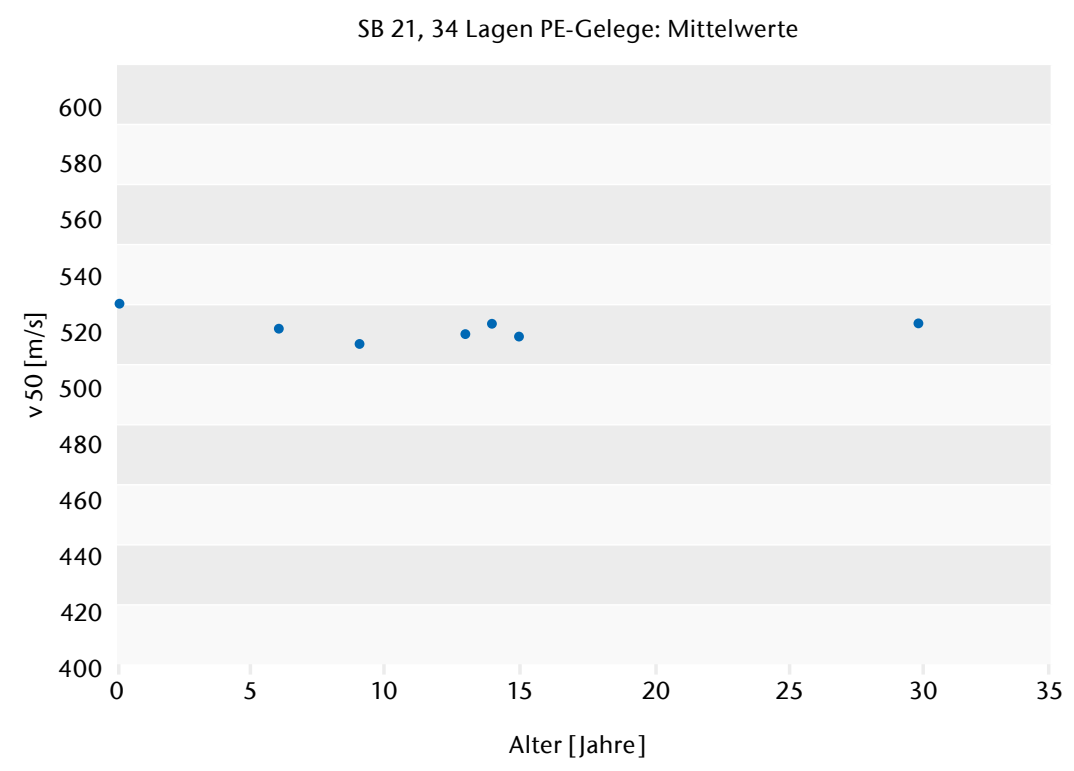

Abb. 2) V50-Werte (Mittelwerte über alle Produktionschargen aus SB21) in Abhängigkeit vom Alter. Die Standardabweichung für die V50-Werte liegt in der Größenordnung 3 bis $5 \mathrm{~m} / \mathrm{s}$.

mustern unterschiedlicher Produktionschargen aus SB21 in Abhängigkeit von ihrem Alter. Die einzelnen Produktionschargen sind farblich unterschiedlich markiert. Die Prüfmuster 19069 sind künstlich gealtert worden (15 und 30 Jahre).

Die Mittelwerte (arithmetisches Mittel) der V50-Grenzgeschwindigkeiten für alle Produktionschargen zusammen genommen zeigt die Abb. 2 .
Zusammenfassend lässt sich feststellen:

1. Die V50-Grenzgeschwindigkeit nimmt mit zunehmendem Alter der Prüflinge de facto nicht ab.

2. Künstliche und natürliche Alterung zeigen sowohl bei den RAL-Werten als auch bei den Beschussversuchen kein signifikant verschiedenes Verhalten - die gewählte künstliche Alterungsmethode bildet (im Zeit- und Scharmittel) die Summe der in- 
dividuellen Belastungen (z. B. Temperatur, Wechselfeuchtigkeit, Tragehäufigkeit und damit einhergehende komplexe mechanische Belastung etc.) in der jeweiligen Westen-Historie gut ab.

\section{Resümee}

Beschussversuche nach STANAG 2920 an vielen Westen einer Produktionscharge und eines Alters mit anschließender Mittelwertbildung liefern sinnvolle Aussagen über die V50Grenzgeschwindigkeit und insbesondere über ausbleibende Änderungen der V50 mit zunehmendem Alter. Im Rahmen dieser Untersuchungen lassen sich mit Hilfe dieser Vorgehensweise bis zu einem simulierten Alter von 30 Jahren keine altersbedingten Änderungen der V50-Grenzgeschwindigkeit bei UHMW-PE DU von DSM Dyneema feststellen.

\subsection{Beschussversuche an Prüfmustern aus SB115}

Wie schon bei den Schutzwesten und Prüfmustern aus SB21 wurden im Rahmen der in diesem Kapitel geschilderten Untersuchungen die Beschusstests nach STANAG 2920 auch an Prüfmustern aus SB115 durchgeführt. Dabei wird das ballistische Paket an maximal 12 fest definierten Positionen auf dem Paket beschossen. Die V50Grenzgeschwindigkeit berechnet sich aus dem arithmetischen Mittel von drei Durch- und drei Steckschüssen, wobei die Differenz zwischen der höchsten und der niedrigsten dieser sechs Geschossgeschwindigkeiten nicht mehr als $40 \mathrm{~m} / \mathrm{s}$ betragen darf. Als weitere Regel kommt hinzu, dass der Durchschuss mit der niedrigsten und der Steckschuss mit der höchsten Geschwindigkeit auf jeden Fall mit in die Berechnung des V50-Wertes einzubeziehen sind.

Die Abb. 3 zeigt den nach STANAG 2920 ermittelten Wert der V50 Grenzgeschwindigkeit für Prüfmuster aus SB115 in Abhängigkeit von ihrem Alter. Die Prüfmuster sind teilweise künstlich gealtert worden (15 und 30 Jahre).

Die Mittelwerte (arithmetisches Mittel) der V50-Grenzgeschwindigkeiten zeigt die Abb. 4.

SB 115, 46 Lagen PE-Gelege: Einzelwerte

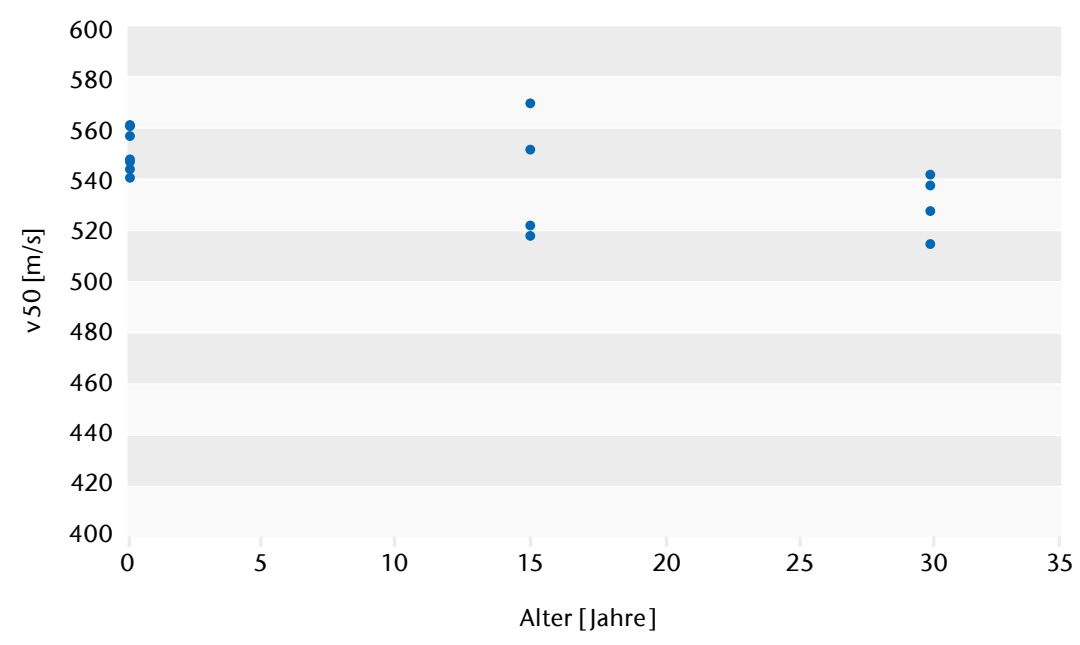

Abb. 3) V50-Werte von Prüfmustern aus SB115 in Abhängigkeit vom Alter. Die Standardabweichung für die V50-Werte liegt in der Größenordnung 10 bis $15 \mathrm{~m} / \mathrm{s}$.

SB 115, 46 Lagen PE-Gelege: Mittelwerte

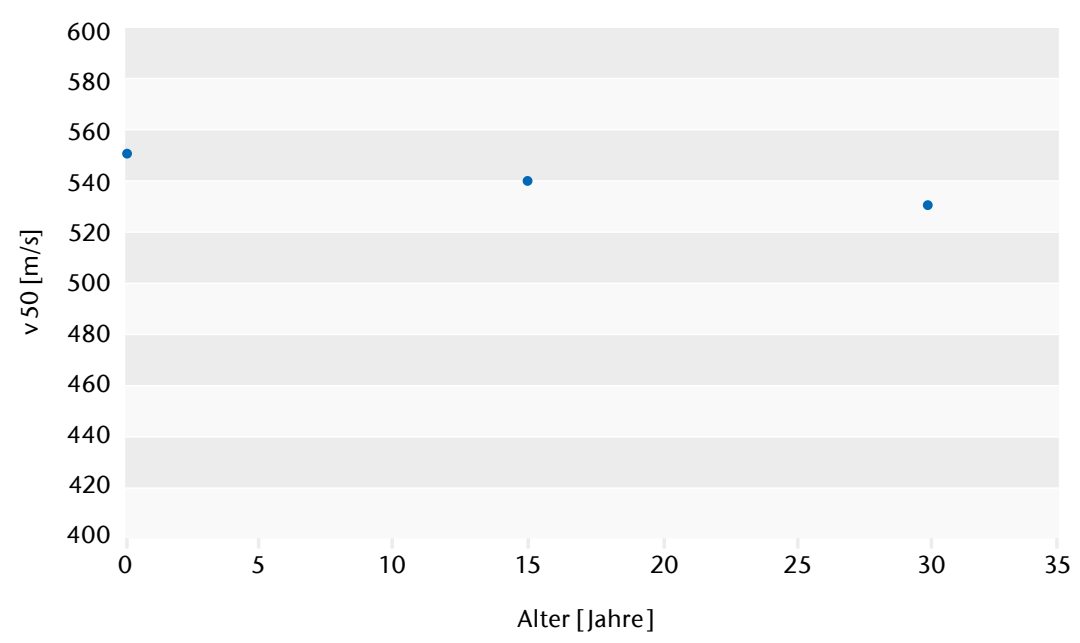

Abb. 4) V50-Werte (Mittelwerte über alle Prüfmuster aus SB115) in Abhängigkeit vom Alter. Die Standardabweichung für die V50-Werte liegt in der Größenordnung 5 bis $7 \mathrm{~m} / \mathrm{s}$.

Zusammenfassend lässt sich feststellen:

Die V50-Grenzgeschwindigkeit nimmt mit zunehmendem Alter der Prüflinge nicht ab (der leichte Abfall über 30 Jahre von $20 \mathrm{~m} / \mathrm{s}$ ist statistisch nicht signifikant).

\section{Resümee}

Beschussversuche nach STANAG 2920 an vielen Westen einer Produktionscharge und eines Alters mit anschließender Mittelwertbildung liefert sinnvolle Aussagen über die V50-Grenzgeschwindigkeit und insbesondere über Änderungen der V50 mit zunehmendem Alter. Im Rahmen dieser Untersuchungen lassen sich mit Hilfe dieser Vorgehensweise bis zu ei- nem simulierten Alter von 30 Jahren keine altersbedingten Änderungen der V50-Grenzgeschwindigkeit bei UHMW-PE DU von DSM Dyneema feststellen.

\section{Zertifizierung von alten Schutz- westen nach SK 1 in Mellrichstadt}

Zertifiziert nach SK 1 wurden ca. 15 Jahre alte Schutzwesten nach der Technischen Richtlinie „Ballistische Schutzwesten“ aus dem Jahr 2000 (Polizeitechnisches Institut der Deutschen Hochschule der Polizei) im Beschussamt Mellrichstadt. Unter anderem wurden die folgenden Ergebnisse erreicht, die insgesamt zu der Zertifi- 
zierung nach SK 1 führten.

1. Entfernung $=10 \mathrm{~m}$, Auftreffwinkel $=90^{\circ}$, Trefferbild TR 2000, 5 Steckschüsse, Temperatur $=21^{\circ} \mathrm{C}$

2. Entfernung $=10 \mathrm{~m}$, Auftreffwinkel $=25^{\circ}$, Trefferbild TR 2000, 3 Steckschüsse, Temperatur $=21^{\circ} \mathrm{C}$

3. Entfernung $=10 \mathrm{~m}$, Auftreffwinkel $=90^{\circ}$, Trefferbild TR 2000, 3 Steckschüsse, Temperatur $=70,0^{\circ} \mathrm{C}$

4. Entfernung $=10 \mathrm{~m}$, Auftreff winkel $=90^{\circ}$, Trefferbild TR 2000, 3 Steckschüsse, Temperatur $=-20^{\circ} \mathrm{C}$

5. Entfernung $=0 \mathrm{~m}$, Auftreffwinkel $=90^{\circ}$, Trefferbild TR 2000, 3 Steckschüsse, Temperatur $=21^{\circ} \mathrm{C}$

6. Grenzwertermittlung, Entfernung $=5 \mathrm{~m}$, Auftreffwinkel $=90^{\circ}$, Prüfungsart $=$ TR $12 / 2003$ Temperatur $=21^{\circ} \mathrm{C}$, $\mathrm{V} 50=512 \mathrm{~m} / \mathrm{s}$, VDelta $=31 \mathrm{~m} / \mathrm{s}$

Die Raumtemperatur betrug bei allen Beschüssen $21^{\circ} \mathrm{C}$ bei einer Luftfeuchtigkeit von 39 \%. Das Geschoss war in jedem Fall die 9 mm-Luger DM 41 CN 36 .

\section{Fazit}

Die Beschussversuche an Schutzwesten bzw. Prüfmustern sowohl aus SB21 als auch aus SB115 zeigen hinsichtlich der V50-Grenzgeschwindigkeit keine nennenswerte Änderung mit zunehmendem Alter. Der V50-Wert von UHWMPE UD, hergestellt von DSM Dyneema, bleibt bis zu einem simulierten Alter von 30 Jahren nahezu konstant. Ältere Schutzwesten bzw. Prüfmuster wurden nicht untersucht.

Im Beschussamt Mellrichstadt wurden ca. 15 Jahre alte Schutzwesten aus SB21 nach SK 1 beschossen. Mit den Ergebnissen von 6 dieser Westenteile wurde eine komplette Zertifizierung nach SK 1 TR2000 durchgeführt.

Insgesamt zeigen weder die von uns durchgeführten Beschussversuche noch die in Mellrichstadt durchgeführte Zertifizierung eine wesentliche Abnahme der ballistischen Schutzeigenschaften mit zunehmendem Probenalter. Die von uns durchgeführten Beschussversuche lassen erkennen, dass die ballistischen Schutzeigenschaften bis zu einem simulierten Alter von 30 Jahren im Wesentlichen erhalten bleiben.

Die Ergebnisse wurden der Fachöffentlichkeit mit einem Vortrag im Rahmen der Fachkonferenz des Polizeitechnischen Instituts der Deutschen Hochschule der Polizei 2017 vorgestellt:

M. Herzog, M. Hendrix: Langzeitverhalten von weichballistischem hochmolekularem Polyethylen, Deutsche Hochschule der Polizei, Fachkonferenz Waffen- und Gerätewesen, 02.03.2017, Nürnberg

\section{LITERATUR}

Chabba S, van Es M, van Klinken EJ, Jongedijk M], Vanek D, Gijsman P, van der Waals ACLM (2007) Accelerated aging study of ultra high molecular weight polyethylene yarn and unidirectional composites for ballistic applications. J Mater Sci 42(8):2891-2893. doi: 10.1007/ s10853-007-1617-7

DSM Dyneema (2017) Personal Armour Applications. https://www.dsm.com/products/dyneema/en_GB/applications/personal-armor.html. Accessed 14 Dec 2017

Frieß R (2006) Prüfung von ballistischem Material für Sonderschutzfahrzeuge. Polizei Verkehr Technik(4):1-4

Geßner E, Hendrix M (2014) Einfluss der Alterung und weiterer externer Faktoren auf die Schutzwirkung von Aramidgeweben. Polizei Verkehr Technik(2):37-40

NATO Standardization Agency (2003) STANAG 2920. Ballistic Test Method for Personal Armour Materials and Combat Clothing, 2nd edn., Brüssel

Polizeitechnisches Institut der Deutschen Hochschule der Polizei (2008) Ballistische Schutzwesten. Technische Richtlinie, Münster

Vereinigung der Prüfstellen für angriffshemmende Materialien und Konstruktionen (2006) Prüfrichtlinie Ballistische Schutzwesten. VPAM BSW 2006, Münster

Polizeitechnisches Institut der Deutschen Hochschule der Polizei (2000) Ballistische Schutzwesten. Technische Richtlinie, Münster

\section{AUTOREN}

Prof. Dr. Michael Hendrix Prof. Dr. Michael Herzog Technische Hochschule Wildau

E-Mail für Korrespondenz: michael.hendrix@th-wildau.de 